\title{
Multi Criterion Decision Making using Intuitionistic Fuzzy Rough Set on Two Universal Sets
}

\author{
T. K. Das ${ }^{1}$, D. P. Acharjya ${ }^{2}$, M. R. Patra ${ }^{3}$ \\ ${ }^{1}$ SITE, VIT University, Vellore, Tamil Nadu, India \\ ${ }^{2}$ SCSE, VIT University, Vellore, Tamil Nadu, India \\ ${ }^{3}$ Dept. of Comp. Sc., Berhampur University, Berhampur, Odisha, India \\ Email: tapan.das@vit.ac.in; dpacharjya@gmail.com; mrpatra12@gmail.com
}

\begin{abstract}
Convergence of information and communication technology has brought a radical change in the way data are collected or generated for ease of multi criterion decision making. The huge data is of no use unless it provides certain information. It is very tedious to select a best option among an array of alternatives. Also, it becomes more tedious when the data contains uncertainties and objectives of evaluation vary in importance and scope. Unlocking the hidden data is of no use to gain insight into customers, markets and organizations. Therefore, processing these data for obtaining decisions is of great challenge. Based on decision theory, in the past many methods are introduced to solve multi criterion decision making problem. The limitation of these approaches is that, they consider only certain information of the weights and decision values to make decisions. Alternatively, it makes less useful when managing uncertain and vague information. In addition, an information system establishes relation between two universal sets. In such situations, multi criterion decision making is very challenging. Therefore, an effort has been made in this paper to process inconsistencies in data with the introduction of intuitionistic fuzzy rough set theory on two universal sets.
\end{abstract}

Index Terms - Rough Set, Solitary Set, Relative Set, Accuracy, Intuitionistic Fuzzy Rough Set, Multi Criteria Decision Making

\section{INTRODUCTION}

The rough sets theory introduced by Pawlak $[1,2]$ is an excellent mathematical tool for the analysis of uncertain, inconsistency and vague description of objects. The rough sets philosophy is based on the assumption that every object of the universe is associated with a certain amount of information. This information in the form of data is expressed by means of some attributes used for object description. Objects having the same description are indiscernible with respect to the available information. The indiscernibility relation thus generated constitutes a mathematical basis of the rough sets theory; it induces a partition of the universe into blocks of indiscernible objects, which can be used to build knowledge about a real or abstract world. The basic idea of rough set is based upon the approximation of sets by pair of sets known as lower approximation and upper approximation. Here, the lower and upper approximation operators are based on equivalence relation. However, in many real life problems, rough set model cannot be applied due to the restrictive condition of requirement of equivalence relation. To this end, rough set is generalized to fuzzy environment such as fuzzy rough set, and rough fuzzy set [3]. Further, the indiscernibility relation is generalized to almost indiscernibility relation with the introduction of fuzzy proximity relation to study many real life problems. Based on fuzzy proximity relation, the concept of rough set on fuzzy approximation spaces is defined [4]. Rough set on fuzzy approximation space is further generalized to rough set on intuitionistic fuzzy approximation space [5]. Different applications in this direction are studied by various authors $[6,7,8]$.

The rough set model is generalized using two distinct and related universal sets by Wong [9]. Further, Guilong Liu [10] defined rough set on two universal sets and fuzzy rough set on two universal sets to study some real life problems. Intuitionistic fuzzy sets [11] extend the concept of the membership functions in fuzzy sets [12] to include the non membership functions and degrees of hesitation. Keeping this in mind, fuzzy rough set on two universal sets is extended to intuitionistic fuzzy rough set on two universal sets by Tripathy and Acharjya [13]. Rough set on two universal sets and intuitionistic fuzzy rough set on two universal sets is widely studied by Tripathy and Acharjya [14] and Acharjya [15].

Multi criterion decision making (MCDM) is a process in which decision makers evaluate each alternative according to multiple criteria. Many representative methods are introduced to solve MCDM problem in business and industry areas. However, a drawback of these approaches is that they mostly consider the decision making with certain information of the weights and decision values. This makes them much less useful when managing uncertain information. To this end, multi criteria fuzzy decision making has been studied in [16, 17]. Several attempts have already been made to use the rough set theory to decision support [18]. The original rough set approach is not able to deal with preference ordered attribute domains like product, quality and market share. For this reason, Greco et al. [19] have proposed an extension of the rough set theory that is able to deal with inconsistencies in multi criteria decision analysis problems. This approach is based on substitution 
of the indiscernibility relation by a dominance relation in the rough approximation of decision classes. But, in many real life problems, an information system establishes relation between two universal sets. Multi criterion decision making on such information system is very challenging. This paper discusses how intuitionistic fuzzy rough set on two universal sets can be employed on MCDM problems for taking decisions.

To unfold the article, the rest of this paper is organized as follows: Section 2 presents a traditional rough set followed by rough set on two universal sets in Section 3 . In Section 4 we present notions and concepts of fuzzy rough set on two universal sets, intuitionistic its algebraic properties, topological classification and measures of uncertainty. Section 4 provides an illustration of the multi criteria decision making (MCDM) problem. Finally, in Section 5, we present conclusion and outlines the directions for future work.

\section{TRADITIONAL ROUGH SET}

Rough set [1] was first put forward and established by Zdzislaw Pawlak (1982) to deal with vague and uncertain data. The basic definition of rough sets is based upon the approximation of a set by a pair of sets known as lower and upper approximation. Let $U$ be the universe of finite non empty set of objects. Let $R \subseteq U \times U$ is an equivalence relation on $U$. The equivalence relation $R$ partitions the set $U$ into disjoint classes and it is denoted as $U / R$. Let $X$ be a subset of $U$. Therefore, the target set $X$ can be described by lower and upper approximation as below, where $\underline{R} X$ and $\bar{R} X$ are $R$-lower and $R$-upper approximations of $X$ respectively.

$$
\begin{aligned}
& \underline{R} X=\cup\left\{X^{\prime} \in U / R: X^{\prime} \subseteq X\right\} \\
& \bar{R} X=\cup\left\{X^{\prime} \in U / R: X^{\prime} \cap X \neq \phi\right\}
\end{aligned}
$$

Boundary region of the set $X, B N_{R}(X)$, is the objects in $X$ that can be distinguished neither as a member nor as a non-member of $X$ employing the relation $R$. It is denoted as $B N_{R}(X)=\bar{R} X-\underline{R} X$. A set $X$ is said to be definable if $\underline{R} X=\bar{R} X$ and the target set is a crisp set i.e., there is no boundary line objects. Similarly, it is said to be rough if $\underline{R} X \neq \bar{R} X$ or equivalently $B N_{R}(X) \neq \phi$.

Rough set has many applications. But, it has certain limitations while handling quantitative information system. It is because quantitative attribute values are not exactly indiscernible rather almost indiscernible. To overcome this limitation rough set has extended to rough set on fuzzy approximation space and rough set on intuitionistic fuzzy approximation space. Similarly, it is not applicable if the information system establishes relation between two universes. For example, relation between hospitals in a city and patients can not be processed in traditional rough set. To overcome this limitation, Liu [10] introduced the concept of rough set on two universal sets. In the next section, we formally state the notions, definitions and concepts of rough set on two universal sets as discussed by Liu.

\section{Rough SET ON Two UNIVERSAL SETS}

An information system provides a means to describe a finite set of objects called a universe with a finite set of attributes thereby represents all the available information and knowledge. But in many real life situations an information system sets up relation with more than one universe. This extends the concept of rough set on single universal set to rough sets on two universal sets. This eventually extends the scope of rough sets to extract knowledge. Liu [10] generalized the rough set models using two distinct universal sets. Let $U$ and $V$ be two universal sets and $R \subseteq(U \times V)$ be a binary relation. A relational system $(U, V, R)$ denotes an approximation space. For an element $x \in U$, right neighborhood or the $R$-relative set of $x$ in $U$ is defined as $r(x)=\cup\{y \in V:(x, y) \in R\}$. Similarly, for an element $y \in V$, the left neighborhood or the $R$-relative set of $y$ in $V$ is defined as $l(y)=\cup\{x \in U:(x, y) \in R\}$.

For any two elements $x_{1}, x_{2} \in U, x_{1}$ and $x_{2}$ are said to be equivalent if $r\left(x_{1}\right)=r\left(x_{2}\right)$. Thus, $\left(x_{1}, x_{2}\right) \in E_{U}$ if and only if $r\left(x_{1}\right)=r\left(x_{2}\right)$, where $E_{U}$ denotes the equivalence relation on $U$. Hence, $E_{U}$ partitions the universal set $\mathrm{U}$ into disjoint subsets. Similarly for any two elements $y_{1}, y_{2} \in V$, we say $y_{1}$ and $y_{2}$ are equivalent if $l\left(y_{1}\right)=l\left(y_{2}\right)$. Thus, $\left(y_{1}, y_{2}\right) \in E_{V}$ if and only if $l\left(y_{1}\right)=l\left(y_{2}\right)$, where $E_{V}$ denotes the equivalence relation on $V$ and partitions the universal set $V$ into disjoint subsets. Therefore for the approximation space $(U, V, R)$, it is clear that $E_{V} \circ R=R=R \circ E_{U}$, where $E_{V} \circ R$ is the composition of $R$ and $E_{V}$. For any $Y \subseteq V$ and the binary relation $R$, we associate two subsets $\underline{R Y}$ and $\bar{R} Y$ called the $R$-lower and $R$-upper approximations of $Y$ respectively, which are given by:

$$
\begin{aligned}
& \underline{R} Y=\cup\{x \in U: r(x) \subseteq Y\} \text { and } \\
& \bar{R} Y=\cup\{x \in U: r(x) \cap Y \neq \phi\}
\end{aligned}
$$

The $R$-boundary of $Y$ is denoted as $B N_{R}(Y)=$ $\bar{R} Y-\underline{R} Y$. The pair $(\bar{R} Y, \underline{R} Y)$ is called as the rough set of $Y \subseteq V$ if $\bar{R} Y \neq \underline{R} Y$ or equivalently $B N_{R}(Y) \neq \phi$. Further, if $U$ and $V$ are finite sets, then the binary relation $R$ from $U$ to $V$ can be represented as $R(x, y)$, where

$$
R(x, y)= \begin{cases}1 & \text { if }(x, y) \in R \\ 0 & \text { if }(x, y) \notin R\end{cases}
$$

The characteristic function of $X \subseteq U$ is defined for each $x \in U$ as follows: 


$$
\chi(x)= \begin{cases}1 & \text { if } x \in X \\ 0 & \text { if } x \notin X\end{cases}
$$

Therefore, the $R$ - lower and $R$ - upper approximations can also be presented in an equivalent form as shown below. Here $\wedge$ and $\vee$ denote the minimum and the maximum operators respectively.

$$
\begin{aligned}
& (\underline{R Y}) x=\underset{y \in V}{\wedge}((1-R(x, y)) \vee Y(y)) \text { and } \\
& (\bar{R} Y) x=\underset{y \in V}{\vee}(R(x, y) \wedge Y(y))
\end{aligned}
$$

Definition 3.1 Let $U$ and $V$ be two universal sets. Let $R$ be a binary relation from $U$ to $V$. If $x \in U$ and $r(x)=\phi$, then we call $x$ is a solitary element with respect to $R$. The set of all solitary elements with respect to the relation $R$ is called as solitary set and is denoted as $S$. Mathematically,

$$
S=\{x \in U: r(x)=\phi\}
$$

\section{FuZZY Rough SET ON Two UNIVERSAL SETS}

The basic idea of rough sets, introduced by Pawlak [1, 2] depends upon the notion of equivalence relations defined over a universe $U$. However, equivalence relations in real life problems are relatively rare in practice. Therefore, efforts have been made to make the relations less significant by removing one or more of the three requirements of an equivalence relation. A fuzzy relation is an extension of the concept of binary relation on any set $U$. Therefore, fuzzy rough sets by Dubois and Prade [3] generalize the concepts of Pawlak rough sets. Further it is generalized to fuzzy rough sets in two universal sets by Liu [10].

Let $U$ be an universe of discourse and $x$ is a particular element of $U$. A fuzzy set $X$ of $U$ is defined as a collection of ordered pairs $\left(x, \mu_{X}(x)\right)$, where $\mu_{X}(x)$ : $U \rightarrow[0,1]$ is a mapping known as the membership function of $X$. The family of all fuzzy sets in $U$ is denoted as $F(U)$. Let $U$ and $V$ be two non empty universal sets. Let $R_{F}$ be a fuzzy binary relation from $U \rightarrow V$. Thus, $\left(U, V, R_{F}\right)$ is called a fuzzy approximation space. For any $Y \in F(V)$ and the fuzzy binary relation $R_{F}$, we associate two subsets $\underline{R_{F} Y}$ and $\overline{R_{F}} Y$ called the $R_{F}$ lower and $R_{F}$-upper approximations of $Y$ respectively. A fuzzy rough set is a pair $\left(R_{F} Y, \overline{R_{F}} Y\right)$ of fuzzy sets on $U$ such that for every $x \in U$

$$
\begin{aligned}
& \left(\underline{R_{F} Y}\right) x=\underset{y \in V}{\wedge}\left(\left(1-\mu_{R_{F}}(x, y)\right) \vee Y(y)\right) \\
& \left(\overline{R_{F}} Y\right) x=\underset{y \in V}{\vee}\left(\mu_{R_{F}}(x, y) \wedge Y(y)\right)
\end{aligned}
$$

Definition 4.1 Let $U$ and $V$ are two universal sets and $R_{F}$ is a fuzzy relation from $U$ to $V$. If $x \in U$ and
$\mu_{R_{F}}(x, y)=0$ for all $y \in V$, then we call $x$ is a solitary element with respect to $R_{F}$. The set of all solitary elements with respect to the fuzzy relation $R_{F}$ is called as solitary set and is denoted as $S$, where

$$
\begin{aligned}
& S=\left\{x: x \in U, \mu_{R_{F}}(x, y)=0 \quad \forall y \in V\right\} \text { and } \\
& \mu_{R_{F}}(x, y)=\operatorname{Minimum}\left(\mu_{U}(x), \mu_{V}(y)\right)
\end{aligned}
$$

The algebraic properties of fuzzy rough set on two universal sets are widely studied by Guilong Liu [10].

\section{INTUITIONISTIC FUZZY ROUGH SET ON TwO UNIVERSAL SETS}

In fuzzy set theory it is taken into consideration that there exist a membership value for all the elements of the set and we do not consider non membership values of the elements of the set. However, it is not true in many real life problems due to the presence of hesitation. In fuzzy set theory, if $\mu(x)$ be the degree of membership of an element $x$, then the degree of non membership of $x$ is calculated using mathematical formula $(1-\mu(x))$ with the assumption that full part of the degree of membership is determinism and in-deterministic part is zero. This is not always applicable in real life and hence intuitionistic fuzzy set theory is better. At the same time, intuitionistic fuzzy set theory reduces to fuzzy set theory if indeterministic part is zero. It indicates that intuitionistic fuzzy set model is a generalized model over fuzzy set model. Therefore, intuitionistic fuzzy rough set on two universal sets is a better model than fuzzy rough set on two universal sets.

Now, we present the definitions, notations and results of intuitionistic fuzzy rough set on two universal sets as introduced by Tripathy and Acharjya [13]. We define the basic concepts leading to intuitionistic fuzzy rough set on two universal sets in which we denote $\mu$ for membership and $v$ for non membership functions that are associated with an intuitionistic fuzzy rough set on two universal sets.

Definition 5.1 [11] Let $U$ be a universe of discourse and $x$ is a particular element of $U$. An intuitionistic fuzzy set $X$ of $U$ is defined as $\left\langle x, \mu_{X}(x), v_{X}(x)\right\rangle$, where the function $\mu_{X}(x): U \rightarrow[0,1]$ and $v_{X}(x): U \rightarrow[0,1]$ define the degree of membership and non membership respectively of the element $x \in U$. For every element $x \in U, 0 \leq \mu_{X}(x)+v_{X}(x) \leq 1$. The amount $1-\left(\mu_{X}(x)\right.$ $\left.+v_{X}(x)\right)$ is called the hesitation part, which may cater either membership value or non membership value or the both. For simplicity we will use $\left(\mu_{X}, v_{X}\right)$ to denote the intuitionistic fuzzy set $X$. The family of all intuitionistic fuzzy subsets of $U$ is denoted by $I F(U)$. The complement of an intuitionistic fuzzy set $X$ is denoted by $X^{\prime}=\left\{<x, v_{X}(x), \mu_{X}(x)>\mid x \in U\right\}$ 
Definition 5.2 [11] Let $U$ and $V$ be two non empty universal sets. An intuitionistic fuzzy relation $R_{I F}$ from $U \rightarrow V$ is an intuitionistic fuzzy set of $(U \times V)$ characterized by the membership function $\mu_{R_{I F}}$ and nonmembership function $v_{R_{I F}}$ where

$$
R_{I F}=\left\{\left\langle(x, y), \mu_{R_{I F}}(x, y), v_{R_{I F}}(x, y)\right\rangle \mid x \in U, y \in V\right\}
$$

with $0 \leq \mu_{R_{I F}}(x, y)+v_{R_{I F}}(x, y) \leq 1$ for every $(x, y) \in$ $U \times V$.

Definition 5.3 [13] Let $U$ and $V$ be two non empty universal sets and $R_{I F}$ is a intuitionistic fuzzy relation from $U$ to $V$. If for $x \in U, \mu_{R_{I F}}(x, y)=0$ and $v_{R_{I F}}(x, y)$ $=1$ for all $y \in V$, then $x$ is said to be a solitary element with respect to $R_{I F}$. The set of all solitary elements with respect to the relation $R_{I F}$ is called the solitary set $S$. That is,

$$
S=\left\{x \mid x \in U, \mu_{R_{I F}}(x, y)=0, v_{R_{I F}}(x, y)=1 \forall y \in V\right\}
$$

Definition 5.4 [13] Let $U$ and $V$ be two non empty universal sets and $R_{I F}$ is a intuitionistic fuzzy relation from $U$ to $V$. Therefore, $\left(U, V, R_{I F}\right)$ is called a intuitionistic fuzzy approximation space. For $Y \in I F(V)$,

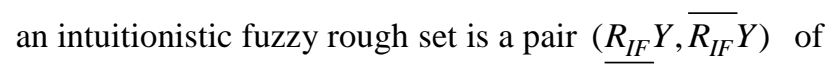
intuitionistic fuzzy set on $U$ such that for every $x \in U$

$$
\begin{aligned}
& \underline{R_{I F} Y}=\left\{\left\langle x, \mu_{\underline{R_{I F}}(Y)}(x), v_{\underline{R_{I F}(Y)}}(x)\right\rangle \mid x \in U\right\} \\
& \overline{R_{I F}} Y=\left\{\left\langle x, \mu_{\overline{R_{I F}}(Y)}(x), v_{\overline{R_{I F}}(Y)}(x)\right\rangle \mid x \in U\right\}
\end{aligned}
$$

where

$$
\begin{aligned}
& \mu_{\underline{R_{I F}}(Y)}(x)=\underset{y \in V}{\wedge}\left[v_{R_{I F}}(x, y) \vee \mu_{Y}(y)\right] \\
& v_{R_{I F}(Y)}(x)=\underset{y \in V}{\vee}\left[\mu_{R_{I F}}(x, y) \wedge v_{Y}(y)\right] \\
& \mu_{\overline{R_{I F}}(Y)}(x)=\underset{y \in V}{\vee}\left[\mu_{R_{I F}}(x, y) \wedge \mu_{Y}(y)\right] \text { and } \\
& v_{\overline{R_{I F}}(Y)}(x)=\underset{y \in V}{\wedge}\left[v_{R_{I F}}(x, y) \vee v_{Y}(y)\right]
\end{aligned}
$$

The pair $\left(\underline{R_{I F}} Y, \overline{R_{I F}} Y\right)$ is called the intuitionistic fuzzy rough set of $Y$ with respect to $\left(U, V, R_{I F}\right)$, where $R_{I F}$, $\overline{R_{I F}}: I F(U) \rightarrow I F(V)$ are referred as lower and upper intuitionistic fuzzy rough approximation operators on two universal sets.

\section{A. Algebraic Properties}

In this section, we discuss the algebraic properties of intuitionistic fuzzy rough set on two universal sets through solitary set as established by Tripathy and Acharjya [13]. These are interesting and valuable in the study of intuitionistic fuzzy rough sets on two universal sets and are useful in finding knowledge from the information system that establishes relation between two universes.

Let $U$ and $V$ be two universal sets. Let $R_{I F}$ be an intuitionistic fuzzy relation from $U$ to $V$ and further let $S$ be the solitary set with respect to $R_{I F}$. Then for $X, Y \in I F(V)$, the following properties holds:

(a) $\underline{R_{I F}}(V)=U$ and $\overline{R_{I F}}(\phi)=\phi$

(b) If $X \subseteq Y$, then $\underline{R_{I F}}(X) \subseteq \underline{R_{I F}}(Y)$ and $\overline{R_{I F}}(X)$ $\subseteq \overline{R_{I F}}(Y)$

(c) $\underline{R_{I F}}(X)=\left(\overline{R_{I F}}\left(X^{\prime}\right)\right)^{\prime}$ and $\overline{R_{I F}}(X)=\left(\underline{R_{I F}}\left(X^{\prime}\right)\right)^{\prime}$

(d) $\overline{R_{I F}} \phi \supseteq S$ and $\overline{R_{I F}} V \subseteq S^{\prime}$, where $S^{\prime}$ denotes the complement of $S$ in $U$.

(e) For any given index set $J$, $X_{i} \in I F(V), \quad \underline{R_{I F}}\left(\bigcup_{i \in J} X_{i}\right) \supseteq \bigcup_{i \in J} \underline{R}_{I F} X_{i} \quad$ and $\overline{R_{I F}}\left(\bigcap_{i \in J} X_{i}\right) \subseteq \bigcap_{i \in J} \overline{R_{I F}} X_{i}$.

$(f)$ For any given index set $J$ $X_{i} \in I F(V), \quad \underline{R_{I F}}\left(\bigcap_{i \in J} X_{i}\right)=\bigcap_{i \in J} R_{I F} X_{i} \quad$ and $\overline{R_{I F}}\left(\bigcup_{i \in J} X_{i}\right)=\bigcup_{i \in J} \overline{R_{I F}} X_{i}$.

\section{B. Approximation of Classification}

In an information system, the understanding of the objects and their attributes influencing the objects with a depicted value are of major concern. The basic philosophy of rough set is based upon the approximation of sets by lower and upper approximation of the set. Here, the lower and upper approximation operators are based on equivalence relation. But, the requirement of an equivalence relation is restrictive and may limit the application of rough set model. Therefore, rough set is generalized by G. Liu [10] to rough set on two universal sets. Further, Acharjya and Tripathy [14] generalized the concepts to intuitionistic fuzzy rough set on two universal sets. Because we are interested in classifications based on intuitionistic fuzzy relation, it is interesting to have the idea of approximation of classifications. It is because classifications of universes play central roles in rough set theory. In this section, we introduce the approximation of classification formally.

Definition 5.5 Let $F=\left\{Y_{1}, Y_{2}, Y_{3}, \cdots, Y_{n}\right\}$, where $n>1$ be a family of non empty sets defined over $V$. We say that $F$ is a classification of $V$ if and only if $\left(Y_{i} \cap Y_{j}\right)=\phi$ for $i \neq j$ and $\sum_{k=1}^{n} Y_{k}=V$.

Definition 5.6 Let $F=\left\{Y_{1}, Y_{2}, Y_{3}, \cdots, Y_{n}\right\}$ be a family of non-empty classification of $V$ and let $R_{I F}$ be a intuitionistic fuzzy relation from $U \rightarrow V$. Then the $R_{I F}$ lower and $R_{I F}$-upper approximation of the family $F$ is given as $\underline{R}_{I F} F=\left\{\underline{R}_{I F} Y_{1}, \underline{R}_{I F} Y_{2}, \underline{R}_{I F} Y_{3}, \cdots, \underline{R}_{I F} Y_{n}\right\}$ and $\bar{R}_{I F} F=\left\{\bar{R}_{I F} Y_{1}, \bar{R}_{I F} Y_{2}, \bar{R}_{I F} Y_{3}, \cdots, \bar{R}_{I F} Y_{n}\right\}$ respectively. 


\section{Measures of Uncertainty}

This section introduces the concept of measures of uncertainty such as accuracy and quality of approximation employing the intuitionistic fuzzy relation $R_{I F}$. We denote the number of objects in a set $V$ by $\operatorname{card}(V)$. Let $F=\left\{Y_{1}, Y_{2}, \cdots, Y_{n}\right\}$ be a family of nonempty classifications. Then the $R_{I F}$-lower and $R_{I F}$-upper approximation of the family $F$ are given as $\underline{R}_{I F} F=$ $\left\{\underline{R}_{I F} Y_{1}, \underline{R}_{I F} Y_{2}, \underline{R}_{I F} Y_{3}, \cdots, \underline{R}_{I F} Y_{n}\right\} \quad$ and $\quad \bar{R}_{I F} F=\left\{\bar{R}_{I F} Y_{1}\right.$, $\left.\bar{R}_{I F} Y_{2}, \bar{R}_{I F} Y_{3}, \cdots, \bar{R}_{I F} Y_{n}\right\}$ respectively. Now we define accuracy of approximation and quality of approximation of the family $F$ employing the intuitionistic fuzzy relation $R_{I F}$ as follows:

Definition 5.7 The accuracy of approximation of $F$ that expresses the percentage of possible correct decisions when classifying objects employing the intuitionistic fuzzy relation $R_{I F}$ is defined as

$$
\alpha_{R_{I F}}(F)=\frac{\sum \operatorname{card}\left(\underline{R}_{I F} Y_{i}\right)}{\sum \operatorname{card}\left(\overline{\bar{R}}_{I F} Y_{i}\right)} \text { for } i=1,2,3, \cdots, n
$$

Definition 5.8 The quality of approximation of $F$ that expresses the percentage of objects which can be correctly classified to classes of $F$ by the intuitionistic fuzzy relation $R_{I F}$ is defined as

$$
v_{R_{I F}}(F)=\frac{\sum \operatorname{card}\left(\underline{R}_{I F} Y_{i}\right)}{\operatorname{card}(V)} \text { for } i=1,2,3, \cdots, n
$$

Definition 5.9 We say that $F=\left\{Y_{1}, Y_{2}, \cdots, Y_{n}\right\}$ is $R_{I F}$ definable if and only if $\underline{R}_{I F}=\bar{R}_{I F}$; that is $\underline{R}_{I F} Y_{i}=\bar{R}_{I F} Y_{i}$ for $i=1,2,3, \cdots, n$.

\section{AN APPLICATION TO MULTI CRITERION DECISION MAKING}

In this section, we depict a real life application of intuitionistic fuzzy rough set on two universal sets to multi criterion decision making. The model application is explained as intuitionistic fuzzy rough set upper approximation. Let us consider the multi criteria decision making in the case of supermarkets in a particular city. However, it is observed that due to several factors such as best possible value for customer's money, quality of the product, style and the behaviour of supporting staff, customers depend on more than one supermarket. Therefore, from customer behaviour it is clear that they are not happy with one supermarket. Hence, intuitionistic fuzzy relation better depicts the relation between the customers and supermarkets.

Let us set the criteria $V=\left\{y_{1}, y_{2}, y_{3}, y_{4}, y_{5}, y_{6}\right\}$, in which $y_{1}$ denotes best possible value of customer's money; $y_{2}$ denotes quality of the product; $y_{3}$ denotes the behavior of supporting staff; $y_{4}$ denotes the location of supermarket; $y_{5}$ denotes availability of items; and $y_{6}$ denotes offers. Let us consider the decisions $U=\left\{d_{1}, d_{2}\right.$, $\left.d_{3}, d_{4}, d_{5}\right\}$, in which $d_{1}$ denotes outstanding; $d_{2}$ denotes most welcome; $d_{3}$ denotes more welcome; $d_{4}$ denotes much welcome; and $d_{5}$ denotes least welcome. Several variety of customers and professionals are invited to the survey that only focuses on the criterion of best possible value of customers money in a supermarket. Therefore, $\left(U, V, R_{I F}\right)$ be an intuitionistic fuzzy approximation space, where $U=\left\{d_{1}, d_{2}, d_{3}, d_{4}, d_{5}\right\}$ and $V=\left\{y_{1}, y_{2}\right.$, $\left.y_{3}, y_{4}, y_{5}, y_{6}\right\}$.

If $15 \%$ people select "outstanding" and $10 \%$ people select "not outstanding"; $25 \%$ select "most welcome" and $20 \%$ select not "most welcome"; $35 \%$ select "more welcome" and 5\% select "not more welcome"; $10 \%$ select "much welcome" and $20 \%$ select "not much welcome"; and 15\% select "least welcome" and $10 \%$ select not "least welcome", then the vector can be obtained as $(0.15,0.1 ; 0.25,0.2 ; 0.35,0.05 ; 0.1,0.2 ; 0.15$, $0.1)^{t}$, where $t$ represents the transpose. Similarly, the decisions based on other criteria are obtained as follows: $(0.1,0.2 ; 0.35,0.21 ; 0.25,0.15 ; 0.1,0.8 ; 0.2,0.7)^{t}, \quad(0.55$, $0.1 ; 0.15,0.45 ; 0.2,0.15 ; 0,0.6 ; 0.1,0.3)^{t},(0.1,0.7 ; 0.1,0.4$; $0.4,0.2 ; 0.2,0.3 ; 0.2,0.1)^{t} \quad, \quad(0,0.6 ; 0,0.5 ; 0.15,0.3 ; 0.35$, $0.1 ; 0.5,0.15)^{t}$, and $(0.25,0.1 ; 0.25,0.2 ; 0.2,0.4 ; 0.1,0.4$; $0.2,0.3)^{t}$. Based on the decision vectors, the intuitionistic fuzzy relation $R_{I F}$ from $U$ to $V$ is presented by the following matrix. We define the intuitionistic fuzzy

\begin{tabular}{|c|c|c|c|c|c|c|}
\hline & $y_{1}$ & $y_{2}$ & $y_{3}$ & $y_{4}$ & $y_{5}$ & $y_{6}$ \\
\hline$d_{1}$ & $0.15,0.1$ & $1,0.2$ & $0.55,0.1$ & $0.1,0.7$ & $0,0.6$ & $0.25,0.1)$ \\
\hline$d_{2}$ & $0.25,0.2$ & $0.35,0.21$ & $0.15,0.45$ & $0.1,0.4$ & $0,0.5$ & $0.25,0.2$ \\
\hline$=d_{3}$ & $0.35,0.05$ & $0.25,0.15$ & $0.2,0.15$ & $0.4,0.2$ & $0.15,0.3$ & $0.2,0.4$ \\
\hline$d_{4}$ & $0.1,0.2$ & $0.1,0.8$ & $0,0.6$ & $0.2,0.3$ & $0.35,0.1$ & $0.1,0.4$ \\
\hline 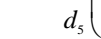 & $0.15,0.1$ & $0.2,0.7$ & $0.1,0.3$ & $0.2,0.1$ & $0.5,0.15$ & $0.2,0.3$ \\
\hline
\end{tabular}
relation $R_{I F} \in I F(U \times V)$ by the following matrix.

It is assumed that there are two categories of customers, where right weights for each criterion in $V$ are $Y_{1}=\left(<d_{1}, 0.35,0.15\right\rangle,\left\langle d_{2}, 0.15,0.3\right\rangle,\left\langle d_{3}, 0.2,0.3\right\rangle,<$ $\left.d_{4}, 0.1,0.5>,<d_{5}, 0.1,0.4>,<d_{6}, 0.1,0.2>\right)$ and $Y_{2}=(<$ $d_{1}, 0.2,0.3>,<d_{2}, 0.4,0.1>,<d_{3}, 0.15,0.4>,<d_{4}, 0.1,0.3$ $>,<d_{5}, 0.1,0.3>,<d_{6}, 0.05,0.5>$ ) respectively. Thus, by using upper approximation we have:

$$
\begin{aligned}
& \mu_{\overline{R_{I F}\left(Y_{1}\right)}}\left(d_{1}\right)=\underset{y \in V}{\vee}\left[\mu_{R_{I F}}\left(d_{1}, y\right) \wedge \mu_{Y_{1}}(y)\right] \\
&=[0.15 \wedge 0.35] \vee[0.1 \wedge 0.15] \vee[0.55 \wedge 0.2] \vee \\
& {[0.1 \wedge 0.1] \vee[0 \wedge 0.1] \vee[0.25 \wedge 0.1] } \\
&= 0.15 \vee 0.1 \vee 0.2 \vee 0.1 \vee 0 \vee 0.1 \\
&= 0.2
\end{aligned}
$$




$$
\begin{aligned}
& v_{\overline{R_{I F}}\left(Y_{1}\right)}\left(d_{1}\right)=\underset{y \in V}{\wedge}\left[v_{R_{I F}}\left(d_{1}, y\right) \vee v_{Y_{1}}(y)\right] \\
& =[0.1 \vee 0.15] \wedge[0.2 \vee 0.3] \wedge[0.1 \vee 0.3] \wedge \\
& {[0.7 \vee 0.5] \wedge[0.6 \vee 0.4] \wedge[0.1 \vee 0.2]} \\
& =0.15 \wedge 0.3 \wedge 0.3 \wedge 0.7 \wedge 0.6 \wedge 0.2 \\
& =0.15 \\
& \mu_{\overline{R_{I F}\left(Y_{1}\right)}}\left(d_{2}\right)=\underset{y \in V}{\vee}\left[\mu_{R_{I F}}\left(d_{2}, y\right) \wedge \mu_{Y_{1}}(y)\right] \\
& =[0.25 \wedge 0.35] \vee[0.35 \wedge 0.15] \vee[0.15 \wedge 0.2] \vee \\
& {[0.1 \wedge 0.1] \vee[0 \wedge 0.1] \vee[0.25 \wedge 0.1]} \\
& =0.25 \vee 0.15 \vee 0.15 \vee 0.1 \vee 0 \vee 0.1 \\
& =0.25 \\
& v_{\overline{R_{I F}}\left(Y_{1}\right)}\left(d_{2}\right)=\underset{y \in V}{\wedge}\left[v_{R_{I F}}\left(d_{2}, y\right) \vee v_{Y_{1}}(y)\right] \\
& =[0.2 \vee 0.15] \wedge[0.21 \vee 0.3] \wedge[0.45 \vee 0.3] \wedge \\
& {[0.4 \vee 0.5] \wedge[0.5 \vee 0.4] \wedge[0.2 \vee 0.2]} \\
& =0.2 \wedge 0.3 \wedge 0.45 \wedge 0.5 \wedge 0.5 \wedge 0.2 \\
& =0.2 \\
& \mu_{\overline{R_{I F}}\left(Y_{1}\right)}\left(d_{3}\right)=\underset{y \in V}{\vee}\left[\mu_{R_{I F}}\left(d_{3}, y\right) \wedge \mu_{Y_{1}}(y)\right] \\
& =[0.35 \wedge 0.35] \vee[0.25 \wedge 0.15] \vee[0.2 \wedge 0.2] \vee \\
& {[0.4 \wedge 0.1] \vee[0.15 \wedge 0.1] \vee[0.2 \wedge 0.1]} \\
& =0.35 \vee 0.15 \vee 0.2 \vee 0.1 \vee 0.1 \vee 0.1 \\
& =0.35 \\
& v_{\overline{R_{I F}}\left(Y_{1}\right)}\left(d_{3}\right)=\underset{y \in V}{\wedge}\left[v_{R_{I F}}\left(d_{3}, y\right) \vee v_{Y_{1}}(y)\right] \\
& =[0.05 \vee 0.15] \wedge[0.15 \vee 0.3] \wedge[0.15 \vee 0.3] \wedge \\
& {[0.2 \vee 0.5] \wedge[0.3 \vee 0.4] \wedge[0.4 \vee 0.2]} \\
& =0.15 \wedge 0.3 \wedge 0.3 \wedge 0.5 \wedge 0.4 \wedge 0.4 \\
& =0.15 \\
& \mu_{\overline{R_{I F}\left(Y_{1}\right)}}\left(d_{4}\right)=\underset{y \in V}{\vee}\left[\mu_{R_{I F}}\left(d_{4}, y\right) \wedge \mu_{Y_{1}}(y)\right] \\
& =[0.1 \wedge 0.35] \vee[0.1 \wedge 0.15] \vee[0 \wedge 0.2] \vee \\
& {[0.2 \wedge 0.1] \vee[0.35 \wedge 0.1] \vee[0.1 \wedge 0.1]} \\
& =0.1 \vee 0.1 \vee 0 \vee 0.1 \vee 0.1 \vee 0.1 \\
& =0.1 \\
& v_{\overline{R_{I F}}\left(Y_{1}\right)}\left(d_{4}\right)=\underset{y \in V}{\wedge}\left[v_{R_{I F}}\left(d_{4}, y\right) \vee v_{Y_{1}}(y)\right] \\
& =[0.2 \vee 0.15] \wedge[0.8 \vee 0.3] \wedge[0.6 \vee 0.3] \wedge \\
& {[0.3 \vee 0.5] \wedge[0.1 \vee 0.4] \wedge[0.4 \vee 0.2]} \\
& =0.2 \wedge 0.8 \wedge 0.6 \wedge 0.5 \wedge 0.4 \wedge 0.4=0.2 \\
& \mu_{\overline{R_{I F}}\left(Y_{1}\right)}\left(d_{5}\right)=\underset{y \in V}{\vee}\left[\mu_{R_{I F}}\left(d_{5}, y\right) \wedge \mu_{Y_{1}}(y)\right] \\
& =[0.15 \wedge 0.35] \vee[0.2 \wedge 0.15] \vee[0.1 \wedge 0.2] \vee \\
& {[0.2 \wedge 0.1] \vee[0.5 \wedge 0.1] \vee[0.2 \wedge 0.1]} \\
& =0.15 \vee 0.15 \vee 0.1 \vee 0.1 \vee 0.1 \vee 0.1 \\
& =0.15 \\
& v_{\overline{R_{I F}}\left(Y_{1}\right)}\left(d_{5}\right)=\underset{y \in V}{\wedge}\left[v_{R_{I F}}\left(d_{5}, y\right) \vee v_{Y_{1}}(y)\right] \\
& =[0.1 \vee 0.15] \wedge[0.7 \vee 0.3] \wedge[0.3 \vee 0.3] \wedge \\
& {[0.1 \vee 0.5] \wedge[0.15 \vee 0.4] \wedge[0.3 \vee 0.2]} \\
& =0.15 \wedge 0.7 \wedge 0.3 \wedge 0.5 \wedge 0.4 \wedge 0.3 \\
& =0.15
\end{aligned}
$$

Hence, the upper approximation for $Y_{1}$ is given as:

$$
\begin{aligned}
& \overline{R_{I F}} Y_{1}=\left\{<d_{1}, 0.2,0.15>,<d_{2}, 0.25,0.2>,\right. \\
& \left.\quad<d_{3}, 0.35,0.15>,<d_{4}, 0.1,0.2>,<d_{5}, 0.15,0.15>\right\}^{t}
\end{aligned}
$$

Similarly, for the set $Y_{2}$ we get the following:

$$
\begin{aligned}
& \mu_{\overline{R_{I F}}\left(Y_{2}\right)}\left(d_{1}\right)=\underset{y \in V}{\vee}\left[\mu_{R_{I F}}\left(d_{1}, y\right) \wedge \mu_{Y_{2}}(y)\right] \\
& =[0.15 \wedge 0.2] \vee[0.1 \wedge 0.4] \vee[0.55 \wedge 0.15] \vee \\
& {[0.1 \wedge 0.1] \vee[0 \wedge 0.1] \vee[0.25 \wedge 0.05]} \\
& =0.15 \vee 0.1 \vee 0.15 \vee 0.1 \vee 0 \vee 0.05 \\
& =0.15 \\
& v_{\overline{R_{I F}}\left(Y_{2}\right)}\left(d_{1}\right)=\wedge_{y \in V}\left[v_{R_{I F}}\left(d_{1}, y\right) \vee v_{Y_{2}}(y)\right] \\
& =[0.1 \vee 0.3] \wedge[0.2 \vee 0.1] \wedge[0.1 \vee 0.4] \wedge \\
& {[0.7 \vee 0.3] \wedge[0.6 \vee 0.3] \wedge[0.1 \vee 0.5]} \\
& =0.3 \wedge 0.2 \wedge 0.4 \wedge 0.7 \wedge 0.6 \wedge 0.5 \\
& =0.2 \\
& \mu_{\overline{R_{I F}}\left(Y_{2}\right)}\left(d_{2}\right)=\underset{y \in V}{\vee}\left[\mu_{R_{I F}}\left(d_{2}, y\right) \wedge \mu_{Y_{2}}(y)\right] \\
& =[0.25 \wedge 0.2] \vee[0.35 \wedge 0.4] \vee[0.15 \wedge 0.15] \vee \\
& {[0.1 \wedge 0.1] \vee[0 \wedge 0.1] \vee[0.25 \wedge 0.05]} \\
& =0.2 \vee 0.35 \vee 0.15 \vee 0.1 \vee 0 \vee 0.05 \\
& =0.35 \\
& v_{\overline{R_{I F}}\left(Y_{2}\right)}\left(d_{2}\right)=\underset{y \in V}{\wedge}\left[v_{R_{I F}}\left(d_{2}, y\right) \vee v_{Y_{2}}(y)\right] \\
& =[0.2 \vee 0.3] \wedge[0.21 \vee 0.1] \wedge[0.45 \vee 0.4] \wedge \\
& {[0.4 \vee 0.3] \wedge[0.5 \vee 0.3] \wedge[0.2 \vee 0.5]} \\
& =0.3 \wedge 0.21 \wedge 0.45 \wedge 0.4 \wedge 0.5 \wedge 0.5 \\
& =0.21 \\
& \mu_{\overline{R_{I F}}\left(Y_{2}\right)}\left(d_{3}\right)=\underset{y \in V}{\vee}\left[\mu_{R_{I F}}\left(d_{3}, y\right) \wedge \mu_{Y_{2}}(y)\right] \\
& =[0.35 \wedge 0.2] \vee[0.25 \wedge 0.4] \vee[0.2 \wedge 0.15] \vee \\
& {[0.4 \wedge 0.1] \vee[0.15 \wedge 0.1] \vee[0.2 \wedge 0.05]} \\
& =0.2 \vee 0.25 \vee 0.15 \vee 0.1 \vee 0.1 \vee 0.05 \\
& =0.25 \\
& v_{\overline{R_{I F}}\left(Y_{2}\right)}\left(d_{3}\right)=\underset{y \in V}{\wedge}\left[v_{R_{I F}}\left(d_{3}, y\right) \vee v_{Y_{2}}(y)\right] \\
& =[0.05 \vee 0.3] \wedge[0.15 \vee 0.1] \wedge[0.15 \vee 0.4] \wedge \\
& {[0.2 \vee 0.3] \wedge[0.3 \vee 0.3] \wedge[0.4 \vee 0.5]} \\
& =0.3 \wedge 0.15 \wedge 0.4 \wedge 0.3 \wedge 0.3 \wedge 0.5=0.15 \\
& \mu_{\overline{R_{I F}}\left(Y_{2}\right)}\left(d_{4}\right)=\underset{y \in V}{\vee}\left[\mu_{R_{I F}}\left(d_{4}, y\right) \wedge \mu_{Y_{2}}(y)\right] \\
& =[0.1 \wedge 0.2] \vee[0.1 \wedge 0.4] \vee[0 \wedge 0.15] \vee \\
& {[0.2 \wedge 0.1] \vee[0.35 \wedge 0.1] \vee[0.1 \wedge 0.05]} \\
& =0.1 \vee 0.1 \vee 0 \vee 0.1 \vee 0.1 \vee 0.05 \\
& =0.1 \\
& v_{\overline{R_{I F}}\left(Y_{2}\right)}\left(d_{4}\right)=\wedge_{y \in V}\left[v_{R_{I F}}\left(d_{4}, y\right) \vee v_{Y_{2}}(y)\right] \\
& =[0.2 \vee 0.3] \wedge[0.8 \vee 0.1] \wedge[0.6 \vee 0.4] \wedge \\
& {[0.3 \vee 0.3] \wedge[0.1 \vee 0.3] \wedge[0.4 \vee 0.5]} \\
& =0.3 \wedge 0.8 \wedge 0.6 \wedge 0.3 \wedge 0.3 \wedge 0.5 \\
& =0.3
\end{aligned}
$$




$$
\begin{aligned}
& \mu_{\overline{R_{I F}}\left(Y_{2}\right)}\left(d_{5}\right)=\underset{y \in V}{\vee}\left[\mu_{R_{I F}}\left(d_{5}, y\right) \wedge \mu_{Y_{2}}(y)\right] \\
&=[0.15 \wedge 0.2] \vee[0.2 \wedge 0.4] \vee[0.1 \wedge 0.15] \vee \\
& {[0.2 \wedge 0.1] \vee[0.5 \wedge 0.1] \vee[0.2 \wedge 0.05] } \\
&= 0.15 \vee 0.2 \vee 0.1 \vee 0.1 \vee 0.1 \vee 0.05 \\
&= 0.2 \\
& v_{\overline{R_{I F}}\left(Y_{2}\right)}\left(d_{5}\right)=\underset{y \in V}{\wedge}\left[v_{R_{I F}}\left(d_{5}, y\right) \vee v_{Y_{2}}(y)\right] \\
&=[0.1 \vee 0.3] \wedge[0.7 \vee 0.1] \wedge[0.3 \vee 0.4] \wedge \\
& \\
& \quad[0.1 \vee 0.3] \wedge[0.15 \vee 0.3] \wedge[0.3 \vee 0.5] \\
&=0.3 \wedge 0.7 \wedge 0.4 \wedge 0.3 \wedge 0.3 \wedge 0.5 \\
&=0.3
\end{aligned}
$$

Hence, the upper approximation for $Y_{2}$ is given as:

$$
\begin{aligned}
& \overline{R_{I F}} Y_{2}=\left\{<d_{1}, 0.15,0.2>,<d_{2}, 0.35,0.21>,\right. \\
& \left.\quad<d_{3}, 0.25,0.15>,<d_{4}, 0.1,0.3>,<d_{5}, 0.2,0.3>\right\}^{t}
\end{aligned}
$$

From the above analysis, according to the principle of maximum membership, the decision for the first category of customers is "more welcome" whereas the decision for the second category of customers is "most welcome".

\section{CONCLUSION}

This paper extends the concepts of intuitionistic fuzzy rough sets on two universal sets further by defining approximation of classifications. We have also defined the accuracy of approximation and quality of approximation of classifications on two universal sets employing intuitionistic fuzzy relation. Multi-criterion decision making employing intuitionistic fuzzy rough set on two universal sets is stressed upon. We have shown how decision changes from one category of customers to another category of customers. The main objective of this paper is to provide the larger audience the concept application of intuitionistic fuzzy rough set on two universal sets. Further research is planned to generalize the study of approximation of classification and measures of uncertainty in the context of intuitionistic fuzzy rough set on two universal sets.

\section{REFERENCES}

[1] Pawlak Z. Rough sets. International Journal of Computer and Information Sciences, 1982, 11, p. 341-356.

[2] Pawlak Z. Rough sets: Theoretical aspects of reasoning about data, Kluwer Academic Publishers, Dordrecht, The Netherlands, 1991.

[3] Dubois D, Prade H. Rough fuzzy sets and fuzzy rough sets. International Journal of General System, 1990, 17, p. 191209.

[4] De S. K. Some aspects of fuzzy sets, rough sets and intuitionistic fuzzy sets, PhD Thesis, IIT-Kharagpur, 1999.

[5] Tripathy B. K. Rough Sets on Intuitionistic Fuzzy Approximation Spaces, In: Proceedings of 3rd International IEEE Conference on Intelligent Systems (IS06), London, 2006, p. 776-779.

[6] Acharjya D. P. and Ezhilarsi L. A knowledge mining model for ranking institutions using rough computing with ordering rules and formal concept analysis, International Journal of Computer Science Issues, 2011, 8(2), p. 417-425.

[7] Acharjya D. P. and Bhattacharjee D. A rough computing based performance evaluation approach for educational institutions, International Journal of Software Engineering and Its Applications, 2013, 7(4), p. 331-348.

[8] Acharjya D. P., Roy Debasrita and Rahaman Md A. Prediction of missing associations using rough computing and Bayesian classification, International Journal of Intelligent Systems and Applications, 2012, 4(11), p 1-13.

[9] Wong S. K. M., Wang L. S. and Yao Y. Y. Interval structure: a framework for representing uncertain information, In: Proceedings of the 8th Conference on Uncertainty in Artificial Intelligence, 1993, p. 336 -343.

[10] Liu G. L. Rough set theory based on two universal sets and its applications, Knowledge Based Systems, 2010, 23, p.110-115.

[11] Atanasov K. T. Intuitionistic fuzzy sets, Fuzzy Sets and Systems, 1986, 20, p. 87-96.

[12] Zadeh L. A. Fuzzy sets, Information and Control, 1965, 8, p. 338-353.

[13] Acharjya D. P. and Tripathy B. K. Intuitionistic fuzzy rough set on two universal sets and knowledge representation, Mathematical Sciences International Research Journal, 2012, 1(2), p. 584-598.

[14] Tripathy B. K. and Acharjya D. P. Approximation of classification and measures of uncertainty in rough set on two universal sets, International Journal of Advanced Science and Technology, Korea, 2012, 40, p. 77-90.

[15] Acharjya D. P. Rough set on two universal sets and knowledge representation, In: Case Studies in Intelligent Computing: Achievements and Trends (Biju Issac and Nauman Israr, Eds.), CRC Press, USA, 2014, p. 79-107.

[16] Hong D. H. and Choi C. H. Multi-criteria fuzzy decisionmaking problems based on vague set theory, Fuzzy Sets and Systems, 2000,114, p. 103-113.

[17] Chen S. M. and Tan J. M. Handing multi-criteria fuzzy decision-making problems based on vague set theory, Fuzzy Sets and Systems, 1994, 67, p. 163-172.

[18] Pawlak Z. and Slowinski R. Rough set approach to multiattribute decision analysis, European Journal of Operational Research, 1994, 72, p. 443-459.

[19] Greco S., Matarazzo B. and Slowinski R. Rough sets theory for multicriteria decision analysis, European Journal of Operational Research, 2001, p. 129- 147.

\section{Authors' Profiles}

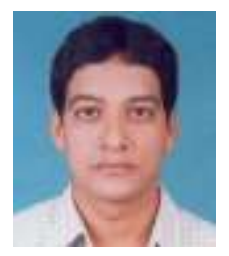

T. K. Das, received his M. Tech. in computer science from Utkal university, India in the year 2003 and M. Sc. from Berhampur University, India in the year 1995. He is currently working as Assistant ProfessorSenior and is pursuing his $\mathrm{Ph}$. D. at VIT University, India. He has authored many international journal and conference papers to his credit. His research interests include Artificial Intelligence, Data Analysis and Data Mining, Databases. He is associated with many professional bodies CSI, and ISCA.

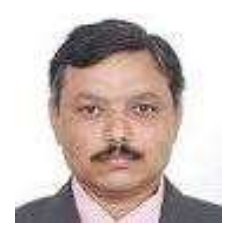

D. P. Acharjya, received his M. Sc. from NIT, Rourkela, India; M. Tech. in computer science from Utkal University, India and obtained his $\mathrm{Ph}$. D. from Berhampur University, India. He has been awarded with Gold Medal in M. Sc. He is presently 
working as a Professor in the School of Computing Sciences and Engineering at VIT University, India. He has authored many international and national papers and four books; Fundamental Approach to Discrete Mathematics, Computer Based on Mathematics, Theory of Computation, and Rough Set in Knowledge Representation and Granular Computing - On Some Aspects to his credit. In addition, he has edited two books with IGI Global, USA. Dr. Acharjya is associated with many professional bodies CSI, ISTE, IMS, AMTI, ISIAM, OITS, IACSIT, CSTA and IAENG.

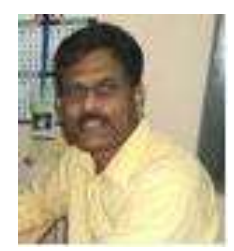

M. R. Patra holds a Ph.D. degree in computer Science from the Central University of Hyderabad. Currently, he is the director of the computer centre at Berhampur University in addition to his teaching assignment in the PG department of computer science. As a United Nations Visiting Fellow, he worked at the International Institute for Software Technology, United Nations University, Macau; and Institute for Development and Research in Banking Technology, Hyderabad. His research interests include service based computing, applications of data mining, and e-Governance. He has supervised $5 \mathrm{Ph} . \mathrm{D}$. students and has more than 100 publications in journals and international conferences to his credit. He is a life member of CSI, ISTE and OITS, and a fellow of ACEEE.

How to cite this paper: T. K. Das, D. P. Acharjya, M. R. Patra,"Multi Criterion Decision Making using Intuitionistic Fuzzy Rough Set on Two Universal Sets", International Journal of Intelligent Systems and Applications (IJISA), vol.7, no.4, pp.26-33, 2015. DOI: 10.5815/ijisa.2015.04.04 